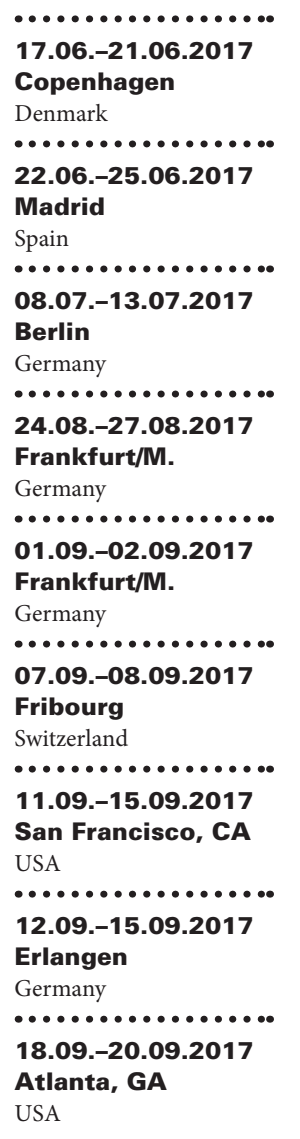

29.09.-03.10.2017

Stuttgart

Germany

•..............

11.10.-13.10.2017

Oldenburg

Germany

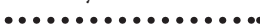

17.10.-20.10.2017

Berlin

Germany

(................

24.10.-27.10.2017

Cologne

Germany [................

25.10.-28.10.2017

Bonn

Germany

-................

21.02.-24.02.2018

Berlin

Germany

[...............

12.09.-14.09.2018

Lübeck

Germany

-....................

29.09.-29.09.2018

Hamburg

Germany 27th Regional Congress of the International Society of Blood Transfusion

22nd Congress of the European Hematology Association (EHA)

63rd Annual Meeting of the International Society on Thrombosis and Haemostasis (ISTH) - Transcending Scientific Boundaries

46th Annual Scientific Meeting of the International Society for Experimental Hematology (ISEH)

\section{Frankfurter Gerinnungssymposium}

Swisstransfusion 2017

43rd Annual Meeting of the American Society for

Histocompatibility and Immunogenetics

47. Jahrestagung der Deutschen Gesellschaft für Immunologie (DGfI)

9th International Conference on Hematology

Jahrestagung der Deutschen, Österreichischen und

Schweizerischen Gesellschaften für Hämatologie und Medizinische Onkologie

14. Jahrestagung der Deutschen Vereinten Gesellschaft für Klinische Chemie und Laboratoriumsmedizin

\section{5th Annual Meeting of the European Society of Gene \& Cell} Therapy

\section{Jahrestagung der Deutschen Gesellschaft für}

Transfusionsmedizin und Immunhämatologie e. V.

26. Jahrestagung der Deutschen Transplantationsgesellschaft

33. Deutscher Krebskongress - Perspektiven verändern Krebs Krebs verändert Perspektiven. Diagnose, Therapie, (Über-)Leben

51. Jahrestagung der Deutschen Gesellschaft für Transfusionsmedizin und Immunhämatologie e. V.
Information: www.isbtweb.org/copenhagen 22nd-congress/key-information/

Information: www.isth2017.org

Information: www.iseh.org/2017Frankfurt

\section{Information:}

www.gerinnungssymposium-frankfurt.de

Information: www.swisstransfusion.ch

Information: www.ashi-hla.org

\section{Information:}

www.dgfi.org/content/47-jahrestagung-der-deutschengesellschaft-f\%C3\%BCr-immunologie

•................................

Information:

www.conferenceseries.com/hematology-meetings

\section{Information:}

www.haematologie-onkologie-2017.com

Information: http://dgkl2017.de/

Information: www.esgct.eu

Information: www.dgti.de

Information: www.dtg2017.de

\section{Information:}

www.krebsgesellschaft.de/deutsche-krebsgesellschaft/

konferenzen/deutscher-krebskongress.html

•..............................

Information: www.dgti.de

Information: www.dtg2018.de

\title{
KARGER
}

